\title{
Occurrence of Gasterophilus spp. in Weanling Foals in Southern Brazil
}

\author{
Rebeca Scalco, Carlos Eduardo Wayne Nogueira ${ }_{\circledast}$ Alice Corrêa Santos, Patrícia Soares Vieira, \\ Nathália Oliveira Ferreira ${ }_{\odot}$, Luciana de Araujo Borba, Lorena Soares Feijó \& Bruna da Rosa Curcio
}

\begin{abstract}
Background: The occurrence of gastrointestinal myiasis caused by Gasterophilus spp. larvae (Diptera: Oestridae) in adult horses has been widely characterized, however data on natural infestation in young foals have been lacking. This observation may be related to the absence of conclusive diagnosis in these individuals, most likely due to logistical or financial constraints. Gastric ulceration is a problem and a significant cause of morbidity in foals, particularly during the weaning stage; therefore, gasterophilosis should be included in the differential diagnosis. The primary purpose of this study was to determine the prevalence of gasterophilosis in 4- to 6-month-old weanling foals.

Materials, Methods \& Results: Seventy-one healthy weanling foals were enrolled in the study. Physical assessment blood sampling and was completed in all foals before the commencement of the experiment. Gastroscopy examinations were performed under sedation (Detomidine $0.01-0.02 \mathrm{mg} / \mathrm{kg}$ ) with a flexible endoscope inserted through nasogastric via. The lumen of the stomach was examined in order to search for botflies' larvae. Following gastroscopy, foals were classified into 2 groups based on the presence of Gasterophilus spp. larvae in their stomachs: 1) Infected and 2) Not Infected. Infected foals received a single dose of commercial trichlorfon and albendazole equine oral gel and were stalled for $24 \mathrm{~h}$. The passed feces were thoroughly examined, searching for elimination of larvae. A total of $64 \%$ of the foals $(n=45 / 71)$ harbored Gasterophilus spp. larvae in the stomach. Mild hyperemia in the gastric mucosa was observed in the attachment sites of the parasites. Physical assessment and hematological parameters' data were analyzed with Shapiro-Wilk normality test. Comparison between groups for clinical signs, hematological parameters and Gasterophilus spp. infection rates were evaluated using the Kruskal-Wallis test or Student's $t$-test. Statistical significance was set at $P<0.05$. There were no statistically significant variations in physical and hematological parameters between foals that were affected and those that were not infected. The larvae found in the feces were subjected to morphological examination, which confirmed the diagnosis of Gasterophilus intestinalis.

Discussion: No systemic clinical indications compatible with Gasterophilus spp. infestation were seen, as described in previous studies of horses infected with the parasite. No botfly eggs were observed in the hair of evaluated foals. During gastroscopy, mild hyperemic lesions in the gastric mucosa were observed in the larvae fixation sites. Although no changes in clinical or hematological parameters were noted, the confirmation of parasite presence is a cause for concern due to horses' tolerance for low infestation levels and poor diagnosis. Additionally, the presence of this myiasis in foals may be a significant stressor during the weaning period and should be included in the differential diagnosis of recurring abdominal pain. Furthermore, infected foals might be a reservoir for the parasite and, contribute to the elimination and spread of the larvae in the environment. Thus, inclusion of young horses in deworming protocols targeted to botfly larvae is needed. This is the first report of Gasterophilus intestinalis myiasis in foals in Brazil. Further research is necessary to fully understand the epidemiology and prevalence of this condition in young horses in Brazil, based on the findings of this study.
\end{abstract}

Keywords: Gasterophilus intestinalis, cavity myiasis, gastroscopy, Oestridae. 


\section{INTRODUCTION}

Gasterophilus spp. belongs to the Oestridae family [12], and its botflies' larvae are common obligatory parasite of the gastrointestinal system of equids. Eight Gasterophilus species have been described globally, with 2 - G. intestinalis and G. nasalis, being reported in Brazil [17]. Gasterophilus spp. causes gastrointestinal myiasis with mild to severe lesions, including stomach and duodenal ulcers, and can lead to anemia, diarrhea, and clinical signs of abdominal pain [8]. Gasterophilus spp. was detected in adult horses in Brazil, typically as a post mortem discovery in the stomach contents of slaughtered or necropsied horses [10].

Gastric ulcers in foals are frequently reported in the literature and are typically related with stressors such as sickness, sudden management changes, extended NSAID medication, early weaning, or transportation [16]. However, the occurrence of Gasterophilus spp. in foals is not commonly described [7]. This observation may be related to the absence of complementary examinations necessary for a definite diagnosis in these individuals, most likely due to logistical or financial constraints. Additional information about gasterophilosis in foals is required, including the species, epidemiology, and prevalence of this parasite, as data on clinical investigation of Gasterophilus spp. infestations in weanling foals are scarce, despite the presence and morphological identification of the botflies. In this context, the purpose of this study was to determine the prevalence of Gasterophilus spp. in weanling foals in southern Brazil and to morphologically identify its species.

\section{MATERIALS AND METHODS}

\section{Animals}

For the purpose of this study, 71 weaned Criollo-crossed foals (4-6 months of age) from the experimental herd of the Veterinary Teaching Hospital of the Federal University of Pelotas (UFPel), Capão do Leão, RS, Brazil were evaluated. The study was conducted from June 2014 to April 2018. Each foal had to be healthy at the beginning of the study and be available for the entire study period. All animals were maintained under the same management: pasture fed, supplemented with commercial pellets and with ad libitum access of water. Before the trial began, all foals underwent physical examination, and blood samples were taken. Body condition was scored as published elsewhere [5].

\section{Sample collection and analysis}

Gastroscopy was performed under sedation with Detomidin ${ }^{1}$ [Detomidine $1 \%$ - $0.02 \mathrm{mg} / \mathrm{kg}$ ] via nasogastric access with a flexible endoscope ${ }^{2}$ [Olympus Optical]. The stomach lumen was examined using previously described methods for detecting botfly larvae. To perform an automated complete blood cell count ${ }^{3}$ [Sysmex pocH-100VTM Hematology-Analyzer], whole blood samples were obtained from each foal immediately prior to the gastroscopy exam in EDTA tubes. The total plasma protein concentration was determined using refractometry, and the fibrinogen concentration was determined using the heat precipitation method.

Thereafter, all foals received a single dose of a commercially available anti-parasitic equine oral gel $^{4}$ [Hipotac $35 \mathrm{mg} / \mathrm{kg}$ triclorphon and $5 \mathrm{mg} / \mathrm{kg}$ albendazole]. Individual dosages were prepared for the appropriate body weight and administered orally according to manufacturer instructions. After deworming, all foals were housed in an individual stall for $24 \mathrm{~h}$. The fecal output was examined, and the larvae found were rinsed in physiological saline solution and fixed in formalin $10 \%$ for subsequent morphological analyses as previous described [17].

\section{Statistical analysis}

According to the presence of Gasterophilus spp. larvae in the stomach, foals were classed as 1) Infected Foals and 2) Not Infected Foals. Physical assessment and hematological parameters were analyzed with Shapiro-Wilk normality test. Comparison between groups for clinical signs, hematological parameters and Gasterophilus spp. infection rates were evaluated using the Kruskal-Wallis test or Student's $t$-test. All statistical analysis were conducted using the software Statistix10, and statistical significance level was set at $P<0.05$.

\section{RESULTS}

All foals presented normal attitude (bright, alert, and responsive) and body condition score $4 / 5$ during physical assessment. All subjects' physical examinations, red blood cell, total nucleated cell, neutrophil and lymphocyte counts, and fibrinogen levels were within the normal reference range, with no statistically significant variations between groups $(P>0.05)$ [Table 1]. 
Thirty-seven weanling foals scoped (64\%) harbored Gasterophilus spp. larvae in the stomach. Mild hyperemia in gastric mucosa was observed in the attachment sites of the parasites (Figure 1). Botfly eggs were not observed on the foals' hair. Nine foals from the infected group (20\%) eliminated larvae in feces within $24 \mathrm{~h}$ after treatment. During morphological analysis, the 3rd stage larvae were identified as Gasterophilus intestinalis (Figure 2), based on the observation of spikes in 2 rows from segments 2 to 8 , where in the spikes of the first row are larger than those of the second.

Table 1. Clinical and hematological features of weanling foals infected by Gasterophilus intestinalis and not infected foals.

\begin{tabular}{ccc}
\hline Parameters & $\begin{array}{c}\text { Infected foals* } \\
(\mathrm{n}=37) \\
(\text { Mean } \pm \mathrm{SD})\end{array}$ & $\begin{array}{c}\text { Not infected foals* } \\
(\mathrm{n}=24) \\
(\text { Mean } \pm \mathrm{SD})\end{array}$ \\
\hline Heart rate $(\mathrm{bpm})$ & $44.40 \pm 2.95$ & $44.0 \pm 6.32$ \\
Respiratory rate $(\mathrm{bpm})$ & $14.00 \pm 2.10$ & $15.20 \pm 4.38$ \\
Temperature $\left({ }^{\circ} \mathrm{C}\right)$ & $38.19 \pm 0.37$ & $37.92 \pm 0.17$ \\
Red blood cells $\left(\mathrm{x} 10^{6} / \mathrm{L}\right)$ & $9.66 \pm 1.65$ & $11.35 \pm 0.78$ \\
Hemoglobin $(\mathrm{g} / \mathrm{L})$ & $11.18 \pm 1.78$ & $11.98 \pm 0.88$ \\
Hematocrit $(\%)$ & $35.01 \pm 5.72$ & $36.16 \pm 6.15$ \\
MCV $(\mathrm{fl})$ & $36.25 \pm 1.03$ & $33.96 \pm 1.02$ \\
MCH $(\mathrm{g} / \mathrm{L})$ & $31.4 \pm 0.65$ & $31.06 \pm 0.72$ \\
Platelets $\left(\mathrm{x} 10^{9} / \mathrm{L}\right)$ & $183.70 \pm 93.78$ & $227.33 \pm 82.08$ \\
Fibrinogen $(\mathrm{mg} / \mathrm{dL})$ & $540.54 \pm 231.86$ & $487.5 \pm 236.84$ \\
Total plasma protein $(\mathrm{g} / \mathrm{dL})$ & $7.66 \pm 0.37$ & $7.38 \pm 0.29$ \\
Total Leukocytes $\left(\mathrm{mm}^{3}\right)$ & $13,819.16 \pm 4,194.79$ & $12,987.08 \pm 3,769.91$ \\
Neutrophils $\left(\mathrm{mm}^{3}\right)$ & $8,431.18 \pm 3,378.19$ & $8,171.58 \pm 2,799.54$ \\
Lymphocytes $\left(\mathrm{mm}^{3}\right)$ & $4,992.24 \pm 2,221.75$ & $4,579.75 \pm 1,502.58$ \\
Monocytes $\left(\mathrm{mm}^{3}\right)$ & $406.40 \pm 384.03$ & $247 \pm 298.74$ \\
Eosinophils $\left(\mathrm{mm}^{3}\right)$ & $210.10 \pm 254.50$ & $451.41 \pm 596.19$ \\
\hline
\end{tabular}

*There was not observed difference between groups $(P>0.05)$. $\mathrm{SD}=$ Standard deviation; ${ }^{1} \mathrm{MCV}=$ Mean corpuscular volume; ${ }^{2} \mathrm{MCH}=$ Mean corpuscular hemoglobin concentration.

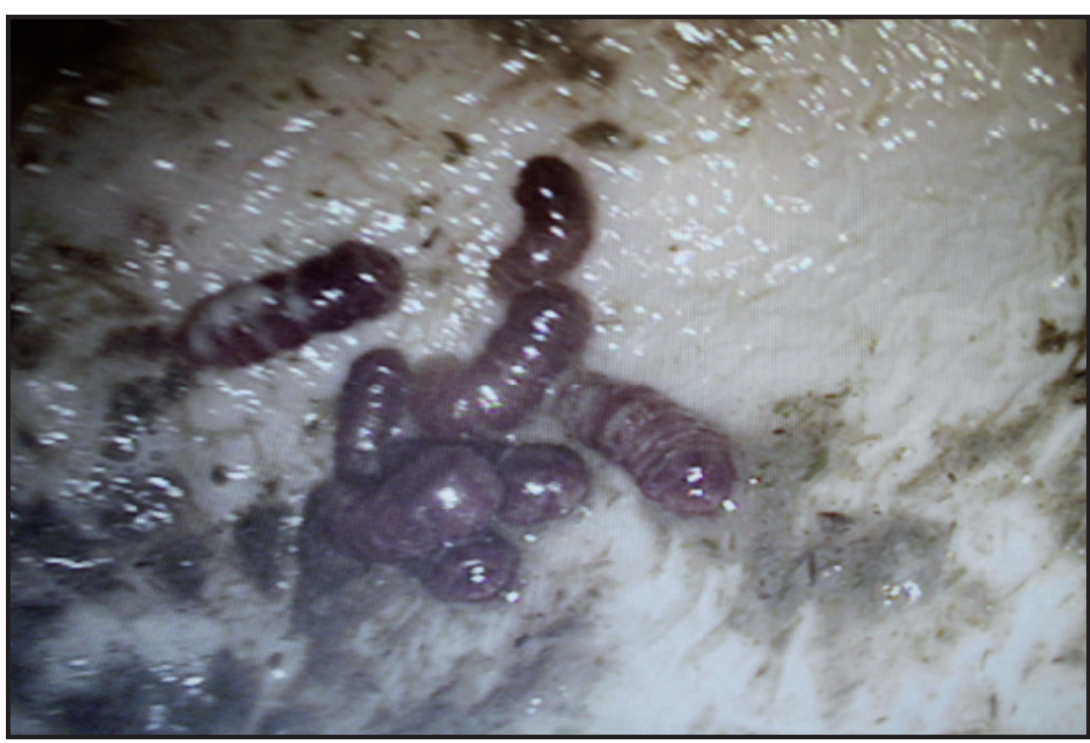

Figure 1. Image of Gasterophilus intestinalis larvae attached to the stomach lumen of a weanling foal. Gastroscopy exam was carried using a flexible endoscope (Olympus) through nasogastric access. 


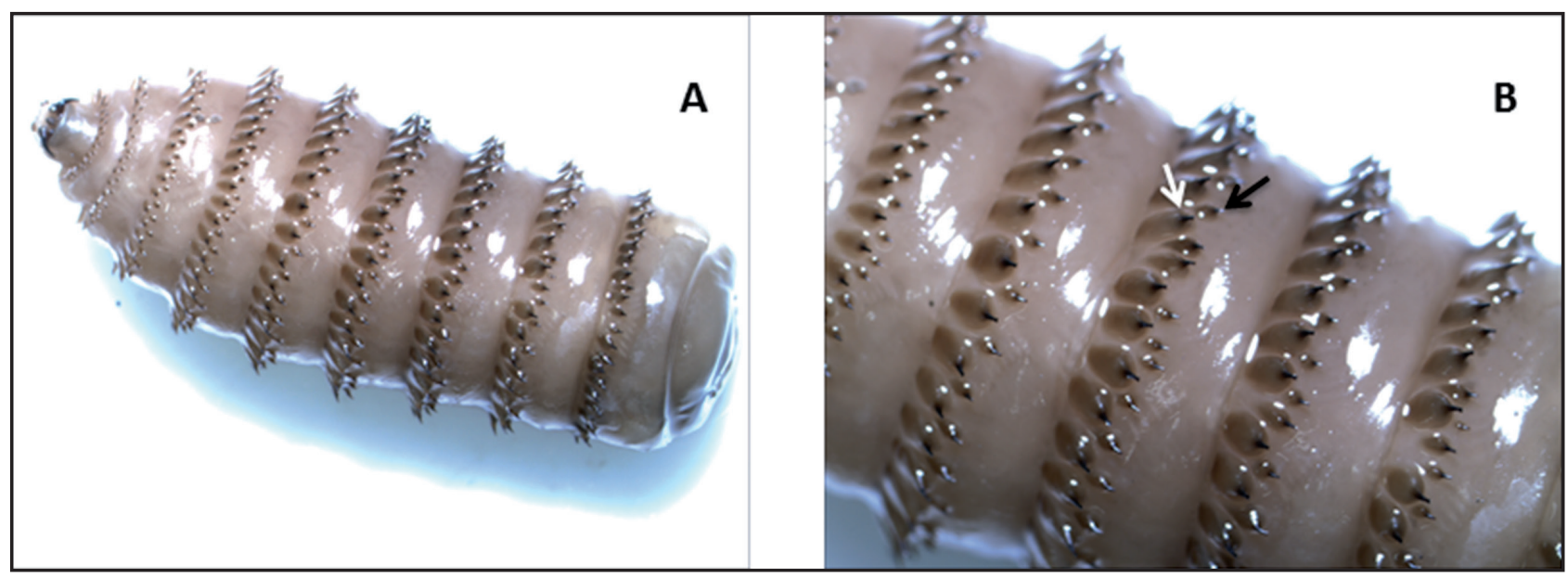

Figure 2. A- Identification of 3rd stage larvae of Gasterophilus intestinalis, using a stereomicroscope [Olympuys SZ61; obj; 10x; zoom 0,67x]. BIdentification was based on the observation of spikes in two rows from segments 2 to 8 , wherein the spines of the first row (white arrow) are larger than those of the second (black arrow).

\section{DISCUSSION}

The current study describes gastrointestinal myiasis in weanling foals caused by Gasterophilus intestinalis. The combination of visualizing the parasite in the foal's stomach during gastroscopy, elimination of larvae in the feces, and morphologically identifying the larvae all contribute to the definitive diagnosis of natural infestation. The presence of the parasite in the stomach lumen was confirmed in 37 of the 71 foals on our study. Elimination of the stage 2 larvae was confirmed in 9 of 71 foals. There were no significant changes in hematological markers reported, despite the presence of a mild level of infestation in all cases.

None of the foals displayed systemic clinical symptoms compatible with Gasterophilus infection. In the hair of assessed foals, no parasite eggs were observed. In the larvae fixation sites, gastroscopy revealed mild hyperemic lesions in the stomach mucosa. However, a heavier infection with bot larvae may result in a more severe reaction on the mucosa of the stomach. Infected horses may have nonspecific clinical indications that may be associated with equine gastric ulceration syndrome (EGUS), gastritis, or colic $[1,9]$. A case of gastro-esophageal reflux in a 5-monthold weanling filly and its association with severe Gasterophilus infestation is described. The foal was presented clinical symptoms such as anorexia, depression, pyrexia and marked bruxism and ptyalism as well as acute abdominal pain. Gastroscopy revealed a dense population of Gasterophilus larvae attached to the lower esophageal sphincter, cardia, and squa- mous mucosa. Throughout hospitalization, the filly received support treatment, parenteral nutrition and was dewormed with ivermectin paste $(200 \mu \mathrm{g} / \mathrm{kg})$ and discharged 10 days after admission [3].

The gastroscopy has proven to be a viable and sensitive tool for identification of Gasterophilus in horse's gastrointestinal tract. A healthy horse's stomach must be clear of parasites and, the mucosal characteristics, while maintaining normal anatomical dimensions being free of lesions, hyperemia, or hyperqueratosis. The equine stomach mucosa is classified histologically into 2 types: glandular and nonglandular. The glandular zone should have a bright pink mucosa, whilst the nonglandular region should be lighter and with prominent folds [6]. Hyperemia in the nonglandular mucosa - the parasite's attachment sites - may be explained by the aggression of the spicules found in the larvae's body, as demonstrated by gastroscopy images.

The prevalence of Gasterophilus in equids can cause serious economic problems worldwide. Gasterophilosis has been linked with clinical signs consistent with severe gastrointestinal lesions in foals $[8,11,13]$. Therefore, the diagnosis of this myiasis in weanling foals is a warning, given the tolerance of horses to low infestations and insufficient diagnosis [10]. Such information must be included amongst the differential diagnosis in recurrent abdominal pain. Furthermore, because gasterophilosis is associated with digestive problems, anemia, and weight loss [4,12,17], an elevated level of infection during the weaning process, which is already stressful, might become a problem. 
Apart from negatively impacting the foal's growth and development, a severe infestation might result in necrotic ulceration, inflammation, glandular tissue loss, fibrosis, and, ultimately, perforation of the gastrointestinal mucosa [2]. Moreover, infected foals might serve as a reservoir for the parasite and contribute to the elimination of the larvae and infection of other horses in the same pasture. Recognizing this, deworming protocols must be adjusted to include medications that specifically target this parasite, as well as fly control in the environment and physical removal of botfly eggs from horses' hair [14,15].

The authors acknowledge that this study's design has limitations. Although the use of macrocylic lactone compounds are the first line anthelmintic drug for treatment of Gasterophilus spp. [15], the use of a commercial association of trichlorphon and albendazole was made attempting to broaden the treatment spectrum. The examination of the foals in different years might have influenced the outcome, as the rate of parasitic infestations can fluctuate with environment and population density. Despite these limitations, our study provides valid information regarding the occurrence of gasterophilosis in young horses.

\section{CONCLUSION}

In conclusion, the present study confirms the occurrence of Gasterophilus intestinalis infestation in weanling foals. While no clinical symptoms of gastric lesions or changes in hematological parameters were identified, endoscopy confirmed the presence of Gasterophilus in the stomachs of 37 of the 71 foals. Unspecific symptoms of gastroduodenal disease can occur in cases when moderate to large number of parasites is present. Adequate environmental management is recommended, as is the inclusion of weanlings in a systematic deparasitation regimen. Further research is necessary to fully understand the epidemiology and prevalence of this parasite in young horses based on the findings of this study.

\section{MANUFACTURERS}

${ }^{1}$ Syntec do Brasil. Barueri, SP, Brazil.

${ }^{2}$ Olympus Optical Brasil Ltda. São Paulo, SP, Brazil.

${ }^{3}$ Sysmex do Brasil Indústria e Comércio Ltda. São José dos Pinhais, PR, Brazil.

${ }^{4}$ Eurofarma. Itapevi, SP, Brazil.

Acknowledgements. Our thanks to the Brazilian funding agencies CAPES, FAPERGS and CNPQ for providing scholarships to the graduate and undergraduate students.

Ethical approval. The study was performed after approval by the Ethics Committee on Animal Experimentation of the UFPel by \#10195/2014. This research did not receive any specific grant from funding agencies in the public, commercial, or not-for-profit sectors.

Declaration of interest. The authors have no competing interests. The authors alone are responsible for the content and writing of the paper.

\section{REFERENCES}

1 Cogley T.P. 1989. Effects of migrating Gasterophilus intestinalis larvae (Diptera: Gasterophilidae) on the mouth of the horse. Veterinary Parasitology. 31(3-4): 317-331. DOI: https://doi.org/10.1016/0304-4017(89)90081-2

2 Deplazes P., Eckert J., Mathis A., Von Samson-Himmelstjerna G. \& Zahner H. 2016. Order Diptera Suborder Brachycera ('short-horn flyes') Family Oestridae. In: Parasitology in Veterinary Medicine. 3rd edn. Wageningen: Wageningen Academic Publishers, pp.489-497.

3 Edens L.M. \& Murray M.J. 1992. Gastro-oesophageal reflux in a weanling filly: association with Gasterophilus spp. infestation. Equine Veterinary Journal. 24(S13): 26-28. DOI: https://doi.org/10.1111/j.2042-3306.1992.tb04785.x

4 Gao D.Z., Liu G.H., Song H.Q, Wang G.L, Wang C.R. \& Zhu X.Q. 2016. The complete mitochondrial genome of Gasterophilus intestinalis, the first representative of the family Gasterophilidae. Parasitology Research. 115: 25732579. DOI: https://doi.org/10.1007/s00436-016-5002-9.

5 Henneke D.R., Potter G.D., Kreider J.L. \& Yeates B.F. 1983. Relationship between condition score, physical measurements, and body fat percentage in mares. Equine Veterinary Journal. 15: 371-372. DOI: https://doi. org/10.1111/j.2042-3306.1983.tb01826.x

6 Herdt T.H \& Sayegh A.I. 2014. Fisiologia do Trato Gastrointestinal In: Cunningham J.G. \& Bradley G.K. (Eds). Tratado de Fisiologia Veterinária. 3.ed. Rio de Janeiro: Elsevier, pp.263-356.

7 Lyons E.T., Tolliver S.C., Collins S.S. \& Drudge J.H. 2001. Transmission of endoparasites in horse foals born on the same pasture on a farm in central Kentucky (1996-1999). Veterinary Parasitology. 97: 113-121. DOI: https://doi. org/10.1016/S0304-4017(01)00393-4 
8 Murray M.J. 1999. Gastroduodenal ulceration in foals. Equine Veterinary Education. 11(4): 199-207. DOI: https:// doi.org/10.1111/j.2042-3292.1999.tb00948.x

9 Otranto D., Milillo P., Capelli G. \& Colwell D.D. 2005. Species composition of Gasterophilus spp. (Diptera, Oestridae) causing equine gastric myiasis in southern Italy: Parasite biodiversity and risks for extinction. Veterinary Parasitology 133: 111-118. DOI: https://doi.org/10.1016/j.vetpar.2005.05.015

10 Rodrigues F.S., Silva C.E., Schmidtt E., Nizoli L.Q., Gotze M.M. \& Silva S.S. 2007. Presence of Gasterophilus (leach, 1817) (díptera: oestridae) in horses in Rio Grande do Sul state, Brazil. Parasitología Latinoamericana. 62(3-4): 122-126. DOI: http://dx.doi.org/10.4067/S0717-77122007000200004

11 Rooney J.R. 1964. Gastric ulceration in foals. Pathology Veterinary. 1(6): 497-503.

12 Sequeira J.L., Tostes R.A. \& Oliveira-Sequeira T.C. 2001. Prevalence and macro- and microscopic lesions produced by Gasterophilus nasalis (Diptera: Oestridae) in the Botucatu Region, SP, Brazil. Veterinary Parasitology. 102: 261266. DOI: https://doi.org/10.1016/S0304-4017(01)00536-2

13 Sweeney H.J. 1991. Gastroduodenal ulceration in foals. Equine Veterinary Education. 3 (2): 80-85.

14 Taylor M.A., Coop R.L. \& Wall R.L. 2016. Parasites of Horses. In: Veterinary Parasitology. 4th edn. Chichester: Wiley- Blackwell, pp.524-561

15 Walden H.S., Ness S.A.L., Mittel L.D., Divers T.J., vaan Laaren K. \& Sellon D.C. 2014. Miscellaneous Parasitic Diseases. In: Equine Infectious Diseases. 2nd edn. Saint Louis: Elsevier, pp.505-514.

16 Weese J.S. 2008. The gastrointestinal system. In: McAuliffe S.B. \& Slovis N.M. (Eds). Color Atlas of Diseases and Disorders of the Foal. Saint Louis: Elsevier, pp.79-131.

17 Zumpt F. 1965. Morphology, Biology and Pathogenesis of Myiasis-producing Flies in Systematic Order - Family: Oestridae. In: Myiasis in man and animals in the Old World: A Textbook for Physicians, Veterinarians, and Zoologists. London: Butterworth, pp.110-128. 